6. H. Lamb, Hydrodynamics, Cambridge, 1924, pp. 341-450.

7. A. Weinstein, Ein hydrodynamicher Unitätssatz, Math. Z. vol. 19 (1924) pp. 265-275.

8. K. FRIEDRICHS, Über ein Minimumproblem für Potentialströmungen mit freien Rande, Math. Ann. vol. 109 (1933) in particular pp. 77-81.

9. T. Carleman, Über eine nichtlineare Randwertaufgabe bei der Gleichung $\Delta u=0$, Math. Z. vol. 9 (1921) pp. 35-43.

10. T. Boggio, Sulle funzioni di variabile complessa ui un'area circolare, Rendiconti della R. Accademia di Torino vol. 47 (1912) pp. 22-37.

11. A. Weinstein, Fluid motion with free boundaries, Proceedings of the First Symposium in Applied Mathematics, American Mathematical Society, 1949, pp. $1-18$.

12. H. Lewy, $A$ note on harmonic functions and a hydrodynamical application, Proc. Amer. Math. Soc. vol. 3 (1952) pp. 111-113.

UNIVERSITY OF MARYLAND

\title{
FUNCTIONS HAVING POSITIVE REAL PART IN AN ELLIPSE
}

W. C. ROYSTER

1. Introduction. Carathéodory [1] proved that if an analytic function $f(z), f(0)=1$, is regular and has positive real part in the unit circle then the $n$th coefficient must lie in the closed circle with center at the origin and with radius equal to 2 , the extremal function being essentially $(1+z)(1-z)^{-1}$. This theorem has been used extensively in the study of various subclasses of univalent functions. It has been generalized to functions which are regular and have positive real part in an annulus by Nehari [2].

In this paper we obtain an analogous theorem for functions which are regular and have positive real part in an ellipse.

2. The main result. Let $f(z)$ be regular in the ellipse $E$ with foci at \pm 1 and semiaxes $a>b, a>1$. Such a function is representable by a series of Tchebychef polynomials which converges uniformly in $E$, $[3 ; 4]$. That is,

$$
f(z)=\sum_{n=0}^{\infty} a_{n} T_{n}(z), \quad z \in E
$$

where $T_{n}(z)=\cos (n \arccos z)$.

$E$ may be represented parametrically by $z=a \cos t+i b \sin t$, $0 \leqq t<2 \pi$. Since $a^{2}-b^{2}=1$ we may write $a=\cosh s, b=\sinh s, s>0$.

Received by the editors January 16, 1958, and in revised form, June 29, 1958. 
Then $z=\cosh s \cos t+i \sinh s \sin t=\cos (t-i s)$ represents the ellipse $E$, where $s=\tanh ^{-1} b / a$. Suppose $a_{0}=1$ and $a_{n}=\alpha_{n}+i \beta_{n}$ then

$$
\begin{aligned}
f(z) & =U(s, t)+i V(s, t)=1+\sum_{n=1}^{\infty} a_{n} T_{n}(\cos (t-i s)) \\
& =1+\sum_{n=1}^{\infty} a_{n} \cos n(t-i s) .
\end{aligned}
$$

Hence

$$
\operatorname{Re}\{f(z)\}=U(s, t)=1+\sum_{n=1}^{\infty} \alpha_{n} \cosh n s \cos n t-\beta_{n} \sinh n s \sin n t
$$

which for fixed $s$ is the Fourier expansion for $U(s, t)$, so that

$$
\begin{gathered}
1=1 / 2 \pi \int_{0}^{2 \pi} U(s, t) d t, \\
\alpha_{n} \cosh n s=1 / \pi \int_{0}^{2 \pi} U(s, t) \cos n t d t, \\
\beta_{n} \sinh n s=1 / \pi \int_{0}^{2 \pi} U(s, t) \sin n t d t .
\end{gathered}
$$

It follows now that

$$
\left|\alpha_{n} \cosh n s+i \beta_{n} \sinh n s\right|=1 / \pi\left|\int_{0}^{2 \pi} U(s, t) e^{-i n t} d t\right| \leqq 2 .
$$

Employing the exponential forms for $\cosh n s$ and $\sinh n s$ (2.4) becomes

$$
\alpha_{n}^{2}\left(R^{n}+R^{-n}\right)^{2}+\beta_{n}^{2}\left(R^{n}-R^{-n}\right)^{2} \leqq 16,
$$

where $R=a+b>1$, which means that $a_{n}=\alpha_{n}+i \beta_{n}$ must be inside the closed ellipse with center at the origin and semiaxes $4\left(R^{n}+R^{-n}\right)^{-1}$ and $4\left(R^{n}-R^{-n}\right)^{-1}$.

We now have the following

THEOREM. Let $f(z)$ be regular and have positive real part in the ellipse $E$ and let $f(z)$ have the expansion

$$
f(z)=1+\sum_{n=1}^{\infty} a_{n} T_{n}(z), \quad z \in E,
$$

in Tchebychef polynomials which converges uniformly in E. Further let $R=a+b>1$ and $a_{n}=\alpha_{n}+i \beta_{n}$, then 


$$
\alpha_{n}^{2}\left(R^{n}+R^{-n}\right)^{2}+\beta_{n}^{2}\left(R^{n}-R^{-n}\right)^{2} \leqq 16 .
$$

This inequality is the best possible.

It remains to show that the inequality $\left(2.5^{\prime}\right)$ is sharp. We shall do this by constructing, for each $n$, a function $f_{n}(z)$ satisfying equality in $\left(2.5^{\prime}\right)$. By the Riemann mapping theorem it is possible to map $E$ on to the right half plane such that an arbitrary point $z_{0}=\cos \left(t_{0}-i s\right)$ on $E$ is mapped into the point at infinity. The mapping function will be uniquely determined up to a linear transformation of the right half plane onto itself. Let $w=f_{n}(z)=F\left(z, z_{0}\right), z_{0}$ on the boundary of $E$, be a function mapping $E$ onto Re $\{w\} \geqq 0$ with $z_{0}$ mapping into the point at infinity. Since $f_{n}(z)$ is analytic in $E$ it has the series expansion $f_{n}(z)=b_{0}+\sum_{n=1}^{\infty} b_{k} T_{k}(z)$.

If $b_{0}=\gamma+i \delta \neq 1$ we consider the function

$$
f_{n}^{*}(z)=\frac{f_{n}(z)-i \delta}{\gamma}=1+\sum_{k=1}^{\infty} a_{k} T_{k}(z)
$$

where $\gamma>0$ by (2.3). $f_{n}^{*}(z)$ has the desired normalization and $\operatorname{Re}\left\{f_{n}^{*}(z)\right\}=0$ almost everywhere on $\Gamma$ the boundary of $E$, hence it suffices to assume that $f_{n}(z)$ has an expansion of the form (2.6).

Suppose we consider the function

$$
Q(z)=1-\left[T_{k}\left(z_{0}\right) T_{k}(z)+U_{k-1}\left(z_{0}\right) U_{k-1}(z)\left(1-z_{0}^{2}\right)^{1 / 2}\left(1-z^{2}\right)^{1 / 2}\right]
$$

where $U_{k-1}(z)=\sin k(t-i s) /\left(1-z^{2}\right)^{1 / 2}$ is a Tchebychef polynomial of the second kind. It is easy to show that $Q(z)$ is non-negative on $\Gamma$ and also that $Q\left(z_{0}\right)=0$. Since $f_{n}(z)$ has a simple pole at $z=z_{0}$ it follows that $f_{n}(z) Q(z)$ is regular on $\Gamma$.

Next consider the integral

$$
I=-1 / \pi \int_{\Gamma} f_{n}(z) Q(z) \frac{d z}{\left(1-z^{2}\right)^{1 / 2}} .
$$

Using the orthogonal properties of the Tchebychef polynomials (i.e., $\int_{\Gamma} T_{m}(z) T_{n}(z)\left(1-z^{2}\right)^{-1 / 2} d z=0,-\pi,-2 \pi$ if $m \neq n, m=n \neq 0$, $m=n=0$, respectively) and $\int_{\Gamma} T_{m}(z) U_{n-1}(z) d z=0$ for all integral $m \geqq 0, n \geqq 1$ we have

$$
\operatorname{Re}\{I\}=\operatorname{Re}\left\{2-T_{n}\left(z_{0}\right) a_{n}\right\} .
$$

Now $\operatorname{Re}\left\{f_{n}(z)\right\}=0$ on $\Gamma$ for $t \neq t_{0}$ and since the integrand in (2.7) is a continuous function of $t$ for $0 \leqq t \leqq 2 \pi$ it follows that the integral vanishes identically. Thus

$$
\operatorname{Re}\left\{T_{n}\left(z_{0}\right) a_{n}\right\}=2
$$


or $\operatorname{Re}\left\{\left(\alpha_{n} \cosh n s+i \beta_{n} \sinh n s\right) e^{i n t_{0}}\right\}=2$ and for a proper choice of $t_{0}$ we have

$$
\left|\alpha_{n} \cosh n s+i \beta_{n} \sinh n s\right|=2
$$

which establishes $\left(2.5^{\prime}\right)$.

We have assumed that $f(z)$ is regular on $\Gamma$ in order to carry out the integration. This assumption may be relinquished by considering the function $f_{\epsilon}(z)=f(\cos (t-i(s-\epsilon)))$ in the ellipse for which $a=\cosh (s-\epsilon), b=\sinh (s-\epsilon)$. If $(2.5)$ is proved for $f_{\epsilon}(z)$ then the corresponding result for $f(z)$ follows by letting $\epsilon \rightarrow 0$.

\section{REFERENCES}

1. C. Carathéodory, Über den Variabilitätsbereich der Fourier'schen Konstanten von Positiven harmonischen Funktionen, Rend. Circ. Mat. Palermo vol. 32 (1911) pp. 193-217.

2. Z. Nehari, Note on positive harmonic functions, J. London Math. Soc. vol. 25 (1950) pp. 19-25.

3. E. Picard, Traite d'Analyse, 3d ed. vol. 2, p. 336.

4. G. Szegö, Orthogonal polynomials, Amer. Math. Soc. Colloquim Publications vol. 23, p. 238.

UNIVERSITY OF KENTUCKY 